\title{
An imputation method for estimating the learning curve in classification problems
}

\author{
Eric B. Laber \\ Kerby Shedden \\ Department of Statistics Department of Statistics \\ North Carolina State University University of Michigan \\ Yang Yang \\ Yahoo! Research Labs \\ June 5, 2018
}




\begin{abstract}
The learning curve expresses the error rate of a predictive modeling procedure as a function of the sample size of the training dataset. It typically is a decreasing, convex function with a positive limiting value. An estimate of the learning curve can be used to assess whether a modeling procedure should be expected to become substantially more accurate if additional training data become available. This article proposes a new procedure for estimating learning curves using imputation. We focus on classification, although the idea is applicable to other predictive modeling settings. Simulation studies indicate that the learning curve can be estimated with useful accuracy for a roughly four-fold increase in the size of the training set relative to the available data, and that the proposed imputation approach outperforms an alternative estimation approach based on parameterizing the learning curve. We illustrate the method with an application that predicts the risk of disease progression for people with chronic lymphocytic leukemia.
\end{abstract}




\section{Introduction}

Predictive models describe the relationship between an outcome and a set of predictor variables, and are widely used in areas ranging from personalized medicine to computational advertising. For example, in personalized medicine the aim may be to predict which patients with a particular disease are likely to respond favorably to a treatment based on information contained in a set of pre-treatment biomarkers (Insel 2009]). Predictive models are developed using a training data set, and their "generalization performance" is typically assessed with respect to a test set that is independent of the training set. Minimizing some measure of the generalization error rate is usually the first priority in predictive modeling, although issues such as model simplicity, interpretability, and ease of implementation may also be important.

One common type of predictive modeling is binary classification, in which the outcome $Y$ takes on one of two possible values. In classification problems, the expected misclassification rate is a natural measure of generalization performance. Suppose we observe a training set of $n$ feature-label pairs $\mathcal{D}_{n}=\left\{\left(X_{i}, Y_{i}\right)\right\}_{i=1}^{n}$ (capital letters such as $X$ and $Y$ denote random variables and lower case letters such as $x$ and $y$ denote instances of these random variables throughout). Using the training data $\mathcal{D}_{n}$, we can construct a classifier $\hat{c}_{n}$, say using logistic regression. The goal is to use the classifier $\hat{c}_{n}$ to accurately predict the labels $Y$ from the observed features $X$ of unlabeled cases. The expected misclassification rate $\tau(n)$ of the classifier $\hat{c}_{n}$ is the expected proportion of incorrectly labeled features $X$ averaged over both the feature-label distribution of test cases and the distribution of $\mathcal{D}_{n}$; that is,

$$
\tau(n) \triangleq \mathbb{E}\left\{Y \neq \hat{c}_{n}(X)\right\}=E_{\mathcal{D}_{n}} E\left\{Y \neq \hat{c}_{n}(X) \mid \mathcal{D}_{n}\right\}
$$

where $\mathbb{E}$ denotes expectation taken with respect to both $(X, Y)$ and the training data $\mathcal{D}_{n}$. Our primary focus here is to estimate the function $\tau(n)$, which has been termed the "learning 
curve" (Amari et al. [1992], Haussler et al. [1996], Hastie et al. [2009] page 243).

Knowledge of the learning curve can contribute to both study design and interpretation of predictive modeling results. Study design questions arise naturally if the training data are acquired in two or more stages, or were obtained from a pilot study with a modest sample size. Such a study may produce encouraging evidence that a useful predictive relationship exists, but one naturally expects that a predictive model obtained from a small training set will not perform as well as one obtained using a larger training set. If we learn that the expected generalization performance of a rule obtained by following the study design employed in our pilot study can be substantially improved by using a larger training set, we would be encouraged to conduct a larger study using the same features and predictive modeling approach.

Learning curves can also contribute to the interpretation of predictive modeling results. In many settings in which predictive modeling is applied, the variables naturally fall into domains. For example, many early genomic studies investigating risk prediction for cancer outcomes used the expression levels of genes associated with cell growth, division, and proliferation to inform the predictions. When considering the performance of these early studies, which often had modest sample sizes, it was natural to ask whether their performance could best be improved by using larger training sets, or by considering additional classes of genes such as those involved in resistance to chemotherapeutic agents or inhibition of the immune response. A similar question arises when considering the integration of data from different domains that may influence disease outcomes, such as environmental influences, measured metabolite levels, and inherited genetic factors.

The rest of the paper is organized as follows. Section 2 reviews some previous work on learning curves. Section 3 describes three approaches to learning curve estimation, including one existing approach and two new approaches. Section 4 compares the performances of these approaches using several simulated examples. Section 5 illustrates the imputation approach 
using a data set in which the goal is to predict the risk that a patient with chronic lymphocytic leukemia (CLL) will experience a poor outcome. Section 6 provides some concluding remarks.

\section{Learning curves}

Learning curves have been an object of interest for several decades. Bounds on the learning curve follow from the work of Vapnik and Chervonenkis Vapnik and Chervonenkis 1971. Vapnik 1982). These bounds have the power law form $a+b / m^{\alpha}$, where $\alpha=1 / 2$ holds in the most realistic settings. The bounds are tight if nothing is known about the distribution of $X$ and one takes a worst-case perspective. If information about the distribution of $X$ is available or can be estimated from observed $X$ values, tighter bounds can be obtained (e.g. Haussler et al. [1996]).

The problem we consider here is to estimate the learning curve rather than to bound it. Thus we consider the setting in which data on $(X, Y)$ are available, on which the estimation can be based, and we focus on traditional criteria for statistical estimation such as bias and variance, rather than on obtaining bounds. To succeed at this estimation, we must capture the general form of the function (e.g. the order at which the function $\tau$ changes with $n$ ), but also the relevant constants and lower order terms.

Our focus here is on binary classification, but for comparison we briefly consider the setting of linear regression using least-squares methods. In this case, an expression for the learning curve $\tau(n)$ can be derived explicitly. The generalization performance in this case is naturally assessed using the mean-square prediction error (MSPE) $E\left[(Y-\hat{Y})^{2}\right]$ for a prediction $\hat{Y}=\hat{Y}(x)$ of the unobserved $Y$ given its feature vector $X=x$. For training sets of sample size $n$, the expected MSPE is $\sigma^{2}(1+\operatorname{tr}(C M) / n)$, where $M=E\left[\left(X^{\prime} X / n\right)^{-1}\right]$ for the training design matrix $X$ and $C=E\left[x^{*} x^{* \prime}\right]$ for the test set covariate vector $x^{*}$. The

reduction in MSPE due to the use of a larger training set is reflected in the term $\sigma^{2} c / n$, 
where $c=\operatorname{tr}(C M)$ captures both the complexity of the model and the similarity of the training and testing distributions of covariate vectors. We note that on the more natural scale RMSPE $=[\mathrm{MSPE}]^{1 / 2}$, this learning curve would have the form $a+b / n^{1 / 2}$.

\section{Approaches to learning curve estimation}

In this section, we describe three approaches to learning curve estimation. The first approach follows a proposal of Mukherjee et al. [2003]. The second and third approaches are new, to our knowledge.

\subsection{Estimating the learning curve via subsampling and extrapo- lation}

In 2003, Mukherjee et al. described an approach to learning curve estimation based on parameterizing the learning curve. We are not aware that a name has been given to this method, and therefore we termed it "SUBEX" for "subsampling and extrapolation." The method parameterizes the learning curve as an inverse power law of the form $\tau(m)=a+$ $b m^{-\alpha}$. As noted above, this expression is exact in the case of linear least squares regression, but may be inexact in the case of classification using logistic regression. The unknown parameters in this expression are $a \in \mathbb{R}$ and $b, \alpha \geq 0$. This parametric form is fit by first using cross-validation on subsamples of the data of various sizes $m^{\prime}<m$ to obtain direct estimates of $\tau\left(m^{\prime}\right)$. Specifically, for a given $m^{\prime}<m$, we can subsample $B$ subsets of the training data of size $m^{\prime}$, fit the classification model to each subset, and use the complementary $m-m^{\prime}$ samples to unbiasedly estimate the error rate. These $B$ error rate estimates can be averaged to estimate $\tau\left(m^{\prime}\right)$. The parametric form for $\tau(m)$ is then fit to these values to estimate $a, b$, and $\alpha$ using some form of nonlinear regression. For example, nonlinear least squares would estimate $a, b$ and $\alpha$ by minimizing 


$$
\sum_{k}\left(\hat{\tau}\left(m_{k}\right)-a-b m_{k}^{-\alpha}\right)^{2}
$$

where the $m_{k} \leq m$ are a set of sample sizes on which the error rate is directly estimated.

As shown in Section 3 , the SUBEX estimator can be positively biased, conveying an overly optimistic assessment of the generalization performance. One reason for this optimism is the asymmetry in the constraints placed on $\tau$. The curve is constrained to be non-increasing, which is quite natural, but owing to the high variance in cross-validation estimators, the constraint is active on a non-negligible proportion of modest-sized training sets under simple generative models. By contrast, aside from being constrained to be non-negative, the curve is unrestricted in how rapidly it can decrease. Thus, when averaged over training sets, a negative bias in the learning curve results.

\subsection{Estimating the learning curve via imputation and interpola- tion}

The second approach we consider for estimating the learning curve uses data imputation and interpolation, hence this approach is termed "IMPINT." In this approach, one first estimates the joint distribution of the feature-label pair $(X, Y)$. This estimation is generally performed by separately estimating the feature distribution $p_{X}$, and the conditional distribution $p_{Y \mid X}$ of the label $Y$ given the feature $X$. After estimating the joint distribution, one can synthesize data sets of any size. The ability to simulate such data sets allows direct estimation of any point on the learning curve. Specifically, one can generate an arbitrary number of training sets of a given size $m$ from $\hat{p}_{X} \hat{p}_{Y \mid X}$, build a classifier on each one, and then average the generalization performance over newly drawn feature-label pairs from $\hat{p}_{X} \hat{p}_{Y \mid X}$. The complete learning curve can be obtained via interpolation among the learning curve points that are directly estimated. 
We now describe in detail the IMPINT procedure based on logistic regression. Suppose we observe a training set $\mathcal{D}_{n}$ of feature-label pairs $\left\{\left(X_{i}, Y_{i}\right)\right\}_{i=1}^{n}$ drawn i.i.d. from an unknown distribution with density $p_{X, Y}(x, y)$. The features $X$ take values in $\mathbb{R}^{p}$, and the binary labels $Y$ are coded to take values in $\{0,1\}$. Define $\pi(x ; \beta) \triangleq \operatorname{logit}\left(x^{\top} \beta\right)=1 /\left(1+e^{-x^{\top} \beta}\right)$. The distribution $p_{X, Y}(x, y)$ factors into the product $p_{Y \mid X}(y \mid x) p_{X}(x)$. Under the logistic regression model the conditional distribution of $Y \mid X$ has the form $p_{Y \mid X}(y \mid x)=\pi\left(x ; \beta^{*}\right)^{y}(1-$ $\left.\pi\left(x ; \beta^{*}\right)\right)^{1-y}$, with $\beta^{*} \in \mathbb{R}^{p}$ denoting the unknown true parameter value. The marginal distribution of the label $X$, denoted by $p_{X}(x)$, is left unspecified for now.

The conditional error rate for a particular training set $\mathcal{D}_{m}$ is

$$
R\left(\mathcal{D}_{m} ; \beta^{*}\right) \triangleq \int\left[\pi\left(x ; \beta^{*}\right) 1\left\{x^{\top} \hat{\beta}_{m} \leq \kappa\right\}+\left(1-\pi\left(x ; \beta^{*}\right)\right) 1\left\{x^{\top} \hat{\beta}_{m}>\kappa\right\}\right] p_{X}(x) d x
$$

where $\hat{\beta}_{m}=\hat{\beta}_{m}\left(\mathcal{D}_{m}\right)$ is the maximum likelihood estimator of $\beta^{*}$ and the classification rule is given by $\hat{c}_{m}(x)=1\left\{x^{\top} \hat{\beta}_{m} \geq \kappa\right\}$ for some threshold $\kappa \in \mathbb{R}$. Using (1), we can express the learning curve as

$$
\tau(m)=\mathbb{E} R\left(\mathcal{D}_{m}, \beta^{*}\right)=\int R\left(\mathcal{D}_{m}, \beta^{*}\right) \prod_{i=1}^{m} p_{X}\left(x_{i}\right) p_{Y \mid X}\left(y_{i}\right) d x_{i} d y_{i} .
$$

If $\beta^{*}$ and $p_{X}(x)$ were both known, one could compute $\tau(m)$ for each $m$ using (3), for example, using Monte Carlo methods to approximate the $2 m+1$-dimensional integral with arbitrary accuracy. More specifically, using $\beta^{*}$ and $p_{X}(x)$ one could generate $B$ training sets $\mathcal{D}_{m}^{(1)}, \mathcal{D}_{m}^{(2)}, \ldots, \mathcal{D}_{m}^{(B)}$, each of size $m$. Fitting a logistic regression model on the $b^{\text {th }}$ training set yields the estimator $\hat{\beta}_{m}^{(b)}$ of $\beta^{*}$. Furthermore, one could use $\beta^{*}$ and $p_{X}(x)$ to generate a large test set $\mathcal{D}_{*}$. For sufficiently large $B$ and $N$, it follows that 


$$
\begin{aligned}
\tau(m) & \approx \frac{1}{B N} \sum_{b=1}^{B} \sum_{(X, Y) \in \mathcal{D}_{*}}\left[\pi\left(X ; \beta^{*}\right) \cdot 1\left\{X^{\boldsymbol{\top}} \hat{\beta}_{m}^{(b)} \leq \kappa\right\}+\left(1-\pi\left(X ; \beta^{*}\right)\right) \cdot 1\left\{X^{\boldsymbol{\top}} \hat{\beta}_{m}^{(b)}>\kappa\right\}\right] \\
& \approx \frac{1}{B N} \sum_{b=1}^{B} \sum_{(X, Y) \in \mathcal{D}_{*}}\left[Y \cdot 1\left\{X^{\boldsymbol{\top}} \hat{\beta}_{m}^{(b)} \leq \kappa\right\}+(1-Y) \cdot 1\left\{X^{\boldsymbol{\top}} \hat{\beta}_{m}^{(b)}>\kappa\right\}\right] .
\end{aligned}
$$

The IMPINT estimate of $\tau(m)$, which we denote by $\hat{\tau}_{I I}(m)$, is formed by applying the approximation given in (4) over imputed training and testing sets generated using an imputation model fit to the complete observed training data $\mathcal{D}_{n}$. More specifically, let $\hat{p}_{X}(x)$ denote an estimator of $p_{X}(x)$ and $\hat{\beta}_{n}$ denote the usual maximum likelihood estimator of $\beta^{*}$. Note that labeled data is not needed to estimate $p_{X}(x)$, hence if additional unlabeled features are available they can be used to improve the estimation of $p_{X}(x)$. The estimators $\hat{p}_{X}(x)$ and $\hat{\beta}_{n}$, substituted for $p_{X}(x)$ and $\beta^{*}$ respectively, can be used to impute $B$ training sets $\hat{\mathcal{D}}_{m}^{(1)}, \hat{\mathcal{D}}_{m}^{(2)}, \ldots, \hat{\mathcal{D}}_{m}^{(B)}$, each of size $m$, and to sample a large synthetic test set $\hat{\mathcal{D}}_{*}$. Using (4), the IMPINT estimator is given by

$$
\hat{\tau}_{I I}(m) \triangleq \frac{1}{B N} \sum_{b=1}^{B} \sum_{(X, Y) \in \hat{\mathcal{D}}_{*}}\left[Y \cdot 1\left\{X^{\top} \hat{\beta}_{m}^{(b)} \leq \kappa\right\}+(1-Y) \cdot 1\left\{X^{\top} \hat{\beta}_{m}^{(b)}>\kappa\right\}\right]
$$

where $\hat{\beta}_{m}^{(b)}=\hat{\beta}_{m}^{(b)}\left(\hat{\mathcal{D}}_{m}^{(b)}\right)$ denotes the maximum likelihood estimator based on the $b^{\text {th }}$ imputed training set $\hat{\mathcal{D}}_{m}^{(b)}$.

The model $\hat{p}_{X}$ for $p_{X}$ can be obtained using any appropriate modeling approach for multivariate data. Some possible approaches are demonstrated in the simulation studies and real data analysis below. To avoid model mis-specification, it is tempting to simply use the empirical distribution function of $X$ in place of $p_{X}$. However, in our experience, this approach does not work well with continuous covariates. In particular, the estimated learning curve tends be substantially biased downward. This may occur because points in 
the training set have positive mass in the testing population. Thus, the model is not relied upon to interpolate probabilities between observed $X$ points; see Efron 1983 for a discussion of the role played by the distance between training and testing sets in classification.

We found that the number of imputed data sets required to ensure that the IMPINT estimate is smoothly non-increasing can be relatively large. As a practical matter, it is more computationally efficient to use a smaller value of $B$ (e.g. $B \approx 500-1000$ ), and then feed the resulting estimate through a monotone smoother (e.g., Friedman and Tibshirani 1984; alternatively a parametric model could be fit as in Mukherjee et al. 2003). We found that the use of a monotone smoother reduces variance without introducing detectable additional bias.

\subsection{Bias reduction for learning curve estimates}

We found that a simple bias reduction substantially improves the performance of the learning estimates obtained from imputed data. This leads to a modified IMPINT approach, which we call BRIE for "Bias Reduced Imputation Estimator." To motivate this approach, consider what happens when we estimate $p_{Y \mid X}$ using the best-fitting regression model (e.g., a fitted logistic regression model). This will overstate the strength of the relationship between $X$ and $Y$, particularly when the true relationship is weak (e.g., if $Y$ and $X$ are independent, then $\hat{p}_{Y \mid X}$ will still exhibit a relationship). Thus, the IMPINT estimator tends to be an optimistic estimator of $\tau(m)$, in the sense that it systematically overstates predictive accuracy. A simple bias correction addresses this problem.

We observed empirically (see section 3) that for any positive integer $k$, the estimator $\hat{\tau}_{I I}(m)-\hat{\tau}_{I I}(m+k)$ exhibits little bias as an estimator of $\tau(m)-\tau(m+k)$. That is, the IMPINT estimator is nearly unbiased for the increments of the learning curve but not necessarily for its overall level. An explanation for this observation parallels the intuition behind the bootstrap as follows. The asymptote of the learning curve $\lim _{m \rightarrow \infty} \tau(m)$ is the 
Bayes error rate and thus depends exclusively on $p_{X}(x)$ and $\beta^{*}$. However, the increments of $\tau$, in addition to depending on $\beta^{*}$ and $p_{X}(x)$, depend on the sampling properties of the estimator $\hat{\beta}_{m}$ as well as the convergence behavior of $\hat{\beta}_{m}$ to its limiting value $\beta^{*}$. The increments of the IMPINT learning curve estimator $\hat{\tau}_{I I}(n)$ are determined by the sampling properties of $\hat{\beta}_{m}^{(b)}$ as an estimator of $\hat{\beta}_{n}$ as well as the manner by which $\hat{\beta}_{m}^{(b)}$ approaches its limiting value. Thus, if the sampling properties of $\hat{\beta}_{m}^{(b)}$ about $\hat{\beta}_{n}$ accurately reflect the sampling properties of $\hat{\beta}_{n}$ about $\beta^{*}$ and if $\hat{p}_{X}(x)$ is a reasonable estimator of $p_{X}(x)$, then it is possible the increments of $\hat{\tau}_{I I}$ approximate the increments of $\tau$.

If one can accurately estimate the increments of the learning curve, all that remains is to find an unbiased estimator of the learning curve at a single training set size. This is provided by the leave-one-out cross-validation (LOOCV) estimator of the expected test error based on the complete observed training set $\mathcal{D}_{n}$, which provides an unbiased estimator of $\tau(n-1)$. Let $\hat{\tau}_{C V}(n-1)$ denote the LOOCV estimator of $\tau(n-1)$. The BRIE is then given by $\hat{\tau}_{B}(m) \triangleq \hat{\tau}_{I I}(m)+\left(\hat{\tau}_{C V}(n-1)-\hat{\tau}_{I I}(n-1)\right)$. Thus, BRIE is simply a shifted version of the IMPINT estimator.

The choice to use the LOOCV estimator of the misclassification rate is not essential. Noting that $\tau(n) \approx \tau(n-1)$, one could employ any unbiased (or nearly unbiased) estimator of the expected test error $\tau(n)$ to recenter the uncorrected estimator of the learning curve. There are also other ways to achieve this bias correction. For instance, the MLE $\hat{\beta}_{n}$ of $\beta$ derived from the training set may be rescaled by a factor $c<1$, producing a shrunken coefficient vector $\beta$. We found that this approach gives similar results to those obtained using the simple additive bias correction. 


\section{Empirical studies}

In this section we examine the performance of the SUBEX, IMPINT, and BRIE procedures in terms of bias and variance, using a series of simulation studies. For each example we used 1000 Monte Carlo iterations, $B=1000$ imputed data sets, and an imputed test set $\mathcal{D}_{*}$ of size 5000. All initial training sets are of size $n=50$ and we estimate $\tau(m)$ for $m=75,100,150$, and 200 using this initial training set. Thus, we are attempting to extrapolate substantially beyond the initial training set size.

For our simulation studies, we consider the following class of models. The distribution of the features $X$ is a $p$-variate normal distribution with isotropic variance-covariance matrix $\Sigma$

given by $\Sigma_{i, j}=r^{|i-j|}$. The true parameter vector $\beta^{*}$ is a $p$-vector of ones. Thus, this class of models is determined by the dimension of the model $p$ and the level of dependence between the features which is governed by the parameter $r \in(-1,1)$.

Figure 1 shows a few examples of learning curves generated using this class of models. The left hand side of Figure 1 shows the learning curve $\tau(m)$ based on training sets of size $n=50$, for $p$ fixed at 15 and $r=0.0,0.25,0.50$, and 0.75 . The figure shows that as $r$ increases, both the learning rate and the asymptotic error rate (i.e. the Bayes error rate) decrease (differences in the learning rate are most evident for sample sizes less than 100). These changes are mostly driven by the fact that as the positive correlation $r$ increases, the distribution of $\left|X^{\top} \beta^{*}\right|$ becomes stochastically larger, and thus fewer points lie near the optimal decision boundary $\left\{x \in \mathbb{R}^{p}: x^{\top} \beta^{*}=0\right\}$. The right hand side of Figure 1 shows the learning curve $\tau(m)$ based on training sets of size $n=50$ for $r$ fixed at 0.10 and $p=10,15,20$, and 25. The figure shows, as would be expected, that the learning curve becomes steeper as the dimension of the problem increases (the raw values of $\tau_{m}$ are difficult to compare across values of $p$ since the Bayes error rate changes with $p$ ). We use these examples to examine bias and variance properties of the BRIE and SUBEX estimator. 

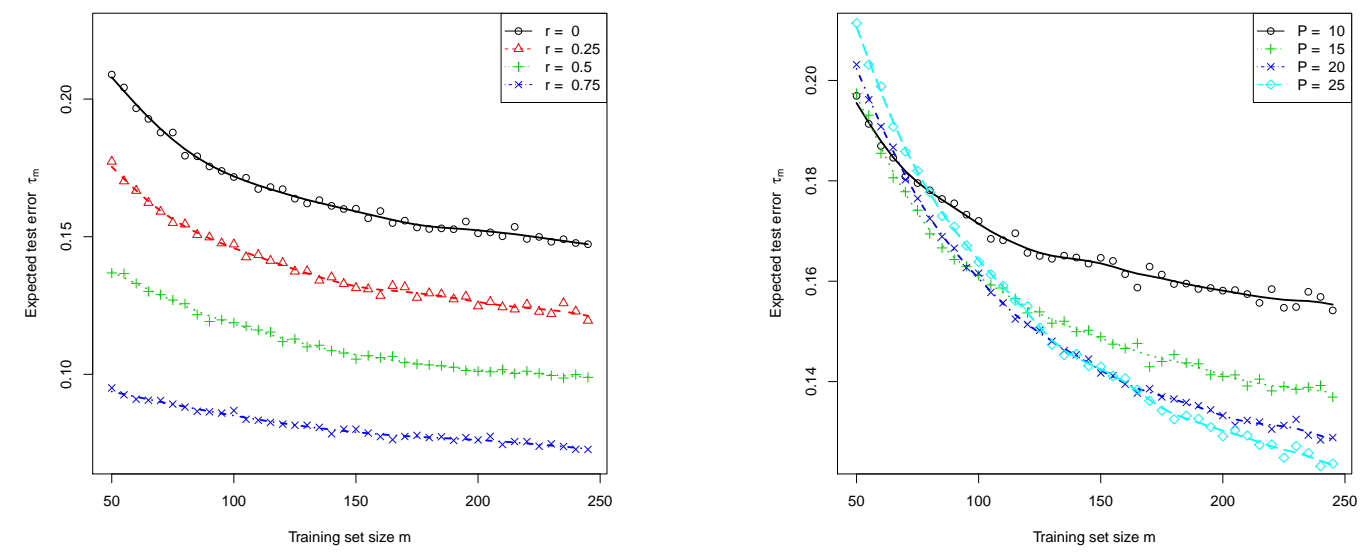

Figure 1: Left: Learning curves $\tau(m)$ for training sets of size $n=50$, for the isotropic normal model with $p=15$ and $r=0, .25, .5, .75$. Right: Learning curves $\tau(m)$ for training sets of size $n=50$ for the isotropic normal model with $r=.10$ and $p=10,15,20,25$.

\subsection{Task one: estimating the improvement in expected error rate}

We first consider the task of estimating the improvement in expected error rate if additional training data are obtained. That is, our goal is to estimate $\delta(n, m) \triangleq \tau(n)-\tau(m)$ for $m \geq n$.

Note that the plug-in BRIE estimate of $\delta(n, m), \hat{\delta}_{B}(n, m) \triangleq \hat{\tau}_{B}(n)-\hat{\tau}_{B}(n)$, is equal to the plug-in IMPINT estimate of $\delta(n, m)$. Estimation of $\delta(n, m)$ is a somewhat easier problem than estimating the entire learning curve $\tau(m)$, as it only requires estimating the shape but not the absolute level of the learning curve. This quantity may be of interest to a researcher who cares more about relative improvement, e.g. a 5\% reduction in expected error rate, than absolute improvement in error rate, e.g. a reduction from $12 \%$ to $7 \%$.

Tables 1 and 2 show the estimated expected values and standard deviations for the BRIE (IMPINT) and SUBEX estimators, on a class of eight models as described at the beginning of this section. The BRIE exhibits substantially smaller bias and standard deviation than the SUBEX estimator in all instances, and provides useful estimates even when extrapolating from $n=50$ to $m=200$, a four-fold increase in sample size. The reduction in variability in 
BRIE relative to SUBEX presumably results from the variance in SUBEX resulting from the use of the LOOCV estimator $\hat{\tau}_{C V}(n-1)$, which is well-known to be highly variable (Toussaint 1974; Efron 1983]; Snapinn and Knoke 1985], Breiman and Spector 1992). The bias in the SUBEX estimator could be due to the true value of $\tau$ not following an inverse power law exactly, or could be due to the asymmetric constraints imposed on the fitted curve, as discussed above.

In tables 1 and 2, the marginal distribution $p_{X}(x)$ was estimated using maximum likelihood for a normal model with unknown mean vector $\mu$ and unknown variance-covariance matrix $\Sigma$ given by $\Sigma_{i, j}=\sigma^{2} \rho^{|i-j|}$. Thus, this simulation provides the BRIE estimator the advantage of knowing the form of the covariance matrix.

Tables 3 and 4 show the analogous results when $\hat{p}_{X}(x)$ is modeled as a multivariate normal distribution with unconstrained variance-covariance matrix. The usual plug-in estimator of the covariance is used. The tables show that the BRIE estimator still exhibits substantially smaller bias and variability than the SUBEX estimator. However, the standard deviation of the BRIE is on average about twice as large compared with using the constrained covariance estimate as in tables 1 and 2 .

\subsection{Task two: estimating the learning curve}

Next we consider the more difficult task of estimating the full learning curve, not just its increments. Our goal is to estimate $\tau(75), \tau(100), \tau(150)$, and $\tau(200)$ using a training set size of $n=50$. As in the previous section, we use the SUBEX estimator as a baseline for comparison.

Tables (5) and (6) show the estimated expected values and standard deviations for the BRIE and the SUBEX estimator of the learning curve on the same eight models considered in the preceding section. Like the results for estimating the improvement in error rate, the BRIE shows only negligible bias for estimating the learning curve, while the bias for 


\begin{tabular}{cc|c|c|c||c|c|c|}
$p$ & $r$ & $\delta(50,75)$ & $\hat{\delta}_{B}(50,75), \mathrm{SD}$ & $\hat{\delta}_{S}(50,75), \mathrm{SD}$ & $\delta(50,100)$ & $\hat{\delta}_{B}(50,100), \mathrm{SD}$ & $\hat{\delta}_{S}(50,100), \mathrm{SD}$ \\
\hline 10 & 0.10 & 0.0173 & $0.0145,0.00477$ & $0.0254,0.0296$ & 0.0249 & $0.0209,0.00613$ & $0.0415,0.0486$ \\
15 & 0.10 & 0.0232 & $0.0253,0.00362$ & $0.0297,0.0323$ & 0.0362 & $0.0376,0.00461$ & $0.0484,0.0531$ \\
20 & 0.10 & 0.0266 & $0.0327,0.00382$ & $0.0383,0.0397$ & 0.0415 & $0.0497,0.00499$ & $0.0625,0.0650$ \\
25 & 0.10 & 0.0294 & $0.0328,0.00347$ & $0.0400,0.0384$ & 0.0475 & $0.0498,0.00450$ & $0.0653,0.0630$ \\
15 & 0.00 & 0.0210 & $0.0263,0.00344$ & $0.0296,0.0321$ & 0.0372 & $0.0390,0.00440$ & $0.0483,0.0527$ \\
15 & 0.25 & 0.0222 & $0.0226,0.00375$ & $0.0272,0.0317$ & 0.0300 & $0.0340,0.00478$ & $0.0444,0.0520$ \\
15 & 0.50 & 0.0100 & $0.0169,0.00462$ & $0.0235,0.0287$ & 0.0180 & $0.0259,0.00615$ & $0.0383,0.0471$ \\
15 & 0.75 & 0.00590 & $0.00986,0.00478$ & $0.0147,0.0166$ & 0.00824 & $0.0151,0.00651$ & $0.0238,0.0271$
\end{tabular}

Table 1: Mean and standard deviation of the BRIE and SUBEX estimator for estimating the improvement in the expected error rate when the training set size is increased to $n=50$ to $m=75$ and $m=100$. The BRIE estimator used a multivariate normal model with the restriction that $\Sigma_{i j}=\sigma^{2} \rho^{|i-j|}$ to estimate $p_{X}(x)$. The BRIE estimator is seen to be significantly less biased than the SUBEX estimator, as well as, possessing smaller standard deviation across training sets.

\begin{tabular}{cc|c|c|c||c|c|c|}
$p$ & $r$ & $\delta(50,150)$ & $\hat{\delta}_{B}(50,150), \mathrm{SD}$ & $\hat{\delta}_{S}(50,150), \mathrm{SD}$ & $\delta(50,200)$ & $\hat{\delta}_{B}(50,200), \mathrm{SD}$ & $\hat{\delta}_{S}(50,200), \mathrm{SD}$ \\
\hline 10 & 0.10 & 0.0322 & $0.0268,0.00679$ & $0.0616,0.0727$ & 0.0388 & $0.0285,0.00686$ & $0.0742,0.0880$ \\
15 & 0.10 & 0.0484 & $0.0497,0.00496$ & $0.0720,0.0794$ & 0.0563 & $0.0556,0.00490$ & $0.0870,0.0963$ \\
20 & 0.10 & 0.0614 & $0.0673,0.00523$ & $0.0930,0.0971$ & 0.0699 & $0.0765,0.00541$ & $0.112,0.117$ \\
25 & 0.10 & 0.0684 & $0.0674,0.00484$ & $0.0973,0.0943$ & 0.0823 & $0.0767,0.00469$ & $0.117,0.124$ \\
15 & 0.00 & 0.0487 & $0.0512,0.00478$ & $0.0719,0.0789$ & 0.0576 & $0.0571,0.00476$ & $0.0868,0.0956$ \\
15 & 0.25 & 0.0459 & $0.0456,0.00506$ & $0.0660,0.0778$ & 0.0525 & $0.0515,0.00487$ & $0.0797,0.0943$ \\
15 & 0.50 & 0.0313 & $0.0356,0.00684$ & $0.0570,0.0705$ & 0.0357 & $0.0410,0.00673$ & $0.0687,0.0855$ \\
15 & 0.75 & 0.0150 & $0.0210,0.00763$ & $0.0351,0.0405$ & 0.0189 & $0.0244,0.00791$ & $0.0421,0.0490$
\end{tabular}

Table 2: Mean and standard deviation of the BRIE and SUBEX estimator for estimating the improvement in the expected error rate when the training set size is increased to $n=50$ to $m=150$ and $m=200$. The BRIE estimator used a multivariate normal model with the restriction that $\Sigma_{i j}=\sigma^{2} \rho^{|i-j|}$ to estimate $p_{X}(x)$. The BRIE estimator is seen to be significantly less biased than the SUBEX estimator, as well as, possessing smaller standard deviation across training sets. 


\begin{tabular}{cc|c|c|c||c|c|c|}
$p$ & $r$ & $\delta(50,75)$ & $\hat{\delta}_{B}(50,75), \mathrm{SD}$ & $\hat{\delta}_{S}(50,75), \mathrm{SD}$ & $\delta(50,100)$ & $\hat{\delta}_{B}(50,100), \mathrm{SD}$ & $\hat{\delta}_{S}(50,100), \mathrm{SD}$ \\
\hline 10 & 0.10 & 0.0173 & $0.0100,0.00206$ & $0.0254,0.0296$ & 0.0249 & $0.0140,0.00330$ & $0.0415,0.0486$ \\
15 & 0.10 & 0.0232 & $0.0209,0.00670$ & $0.0297,0.0323$ & 0.0362 & $0.0314,0.00865$ & $0.0484,0.0531$ \\
20 & 0.10 & 0.0266 & $0.0240,0.00372$ & $0.0383,0.0397$ & 0.0415 & $0.0372,0.00519$ & $0.0625,0.0650$ \\
25 & 0.10 & 0.0294 & $0.0256,0.00748$ & $0.0400,0.0384$ & 0.0475 & $0.0404,0.0102$ & $0.0653,0.0630$ \\
15 & 0.00 & 0.0210 & $0.0217,0.00704$ & $0.0296,0.0321$ & 0.0372 & $0.0325,0.00910$ & $0.0483,0.0527$ \\
15 & 0.25 & 0.0222 & $0.0184,0.00620$ & $0.0272,0.0317$ & 0.0300 & $0.0280,0.00806$ & $0.0444,0.0520$ \\
15 & 0.50 & 0.0100 & $0.0140,0.00568$ & $0.0235,0.0287$ & 0.0180 & $0.0215,0.00750$ & $0.0383,0.0471$ \\
15 & 0.75 & 0.00590 & $0.00879,0.00413$ & $0.0147,0.0166$ & 0.00824 & $0.0135,0.00554$ & $0.0238,0.0271$
\end{tabular}

Table 3: Mean and standard deviation of the BRIE and SUBEX estimator for estimating the improvement in the expected error rate when the training set size is increased to $n=50$ to $m=75$ and $m=100$. The BRIE estimator used an unrestricted multivariate normal model to estimate $p_{X}(x)$. The BRIE estimator is seen to be significantly less biased than the SUBEX estimator, as well as, possessing smaller standard deviation across training sets.

\begin{tabular}{cc|c|c|c||c|c|c|}
$p$ & $r$ & $\delta(50,150)$ & $\hat{\delta}_{B}(50,150), \mathrm{SD}$ & $\hat{\delta}_{S}(50,150), \mathrm{SD}$ & $\delta(50,200)$ & $\hat{\delta}_{B}(50,200), \mathrm{SD}$ & $\hat{\delta}_{S}(50,200), \mathrm{SD}$ \\
\hline 10 & 0.10 & 0.0322 & $0.0202,0.00476$ & $0.0616,0.0727$ & 0.0388 & $0.0240,0.00563$ & $0.0742,0.0880$ \\
15 & 0.10 & 0.0484 & $0.0421,0.00939$ & $0.0720,0.0794$ & 0.0563 & $0.0477,0.00918$ & $0.0870,0.0963$ \\
20 & 0.10 & 0.0614 & $0.0519,0.0101$ & $0.0930,0.0971$ & 0.0699 & $0.0603,0.00998$ & $0.112,0.117$ \\
25 & 0.10 & 0.0684 & $0.0578,0.0118$ & $0.0973,0.0943$ & 0.0823 & $0.0682,0.0118$ & $0.117,0.124$ \\
15 & 0.00 & 0.0487 & $0.0435,0.00991$ & $0.0719,0.0789$ & 0.0576 & $0.0492,0.00974$ & $0.0868,0.0956$ \\
15 & 0.25 & 0.0459 & $0.0381,0.00878$ & $0.0660,0.0778$ & 0.0525 & $0.0436,0.00857$ & $0.0797,0.0943$ \\
15 & 0.50 & 0.313 & $0.0298,0.00839$ & $0.0570,0.0705$ & 0.0357 & $0.0345,0.00836$ & $0.0687,0.0855$ \\
15 & 0.75 & 0.0150 & $0.0188,0.00636$ & $0.0351,0.0405$ & 0.0189 & $0.0218,0.00650$ & $0.0421,0.0490$
\end{tabular}

Table 4: Mean and standard deviation of the BRIE and SUBEX estimator for estimating the improvement in the expected error rate when the training set size is increased to $n=50$ to $m=150$ and $m=200$. The BRIE estimator used an unrestricted multivariate normal model to estimate $p_{X}(x)$. The BRIE estimator is seen to be significantly less biased than the SUBEX estimator, as well as, possessing smaller standard deviation across training sets. 


\begin{tabular}{cc|c|c|c||c|c|c|}
$p$ & $r$ & $\tau(75)$ & $\hat{\tau}_{B}(75), \mathrm{SD}$ & $\hat{\tau}_{S}(75), \mathrm{SD}$ & $\tau(100)$ & $\hat{\tau}_{B}(100), \mathrm{SD}$ & $\hat{\tau}_{S}(100), \mathrm{SD}$ \\
\hline 10 & 0.10 & 0.179 & $0.184,0.0634$ & $0.166,0.0735$ & 0.172 & $0.179,0.0619$ & $0.152,0.0828$ \\
15 & 0.10 & 0.174 & $0.175,0.0636$ & $0.141,0.0755$ & 0.161 & $0.164,0.0639$ & $0.127,0.0832$ \\
20 & 0.10 & 0.176 & $0.177,0.0679$ & $0.154,0.0831$ & 0.161 & $0.163,0.0678$ & $0.136,0.0926$ \\
25 & 0.10 & 0.181 & $0.187,0.0715$ & $0.174,0.0851$ & 0.163 & $0.172,0.0713$ & $0.151,0.0994$ \\
15 & 0.00 & 0.187 & $0.190,0.0655$ & $0.169,0.0831$ & 0.171 & $0.179,0.0664$ & $0.151,0.0940$ \\
15 & 0.25 & 0.155 & $0.158,0.0610$ & $0.147,0.0739$ & 0.147 & $0.148,0.0610$ & $0.134,0.0814$ \\
15 & 0.50 & 0.126 & $0.126,0.0581$ & $0.110,0.0686$ & 0.118 & $0.118,0.0582$ & $0.100,0.0748$ \\
15 & 0.75 & 0.0891 & $0.0861,0.0507$ & $0.0744,0.0505$ & 0.0868 & $0.0814,0.0508$ & $0.0683,0.0527$
\end{tabular}

Table 5: Mean and standard deviation of the BRIE and SUBEX estimator for estimating the learning curve when the training set size is increased from $n=50$ to $m=75$ and $m=100$. The BRIE estimator used a multivariate normal model with the restriction that $\Sigma_{i j}=\sigma^{2} \rho^{|i-j|}$ to estimate $p_{X}(x)$. The BRIE estimator is seen to be significantly less biased than the SUBEX estimator, as well as, possessing smaller standard deviation across training sets.

SUBEX is substantial. BRIE also has a smaller variance than SUBEX, but the advantage is substantially smaller than in the case of estimating learning curve increments. This is likely due to the fact that the bias correction used in the BRIE method is based on the highly variable leave-one-out cross validation estimator of $\tau(n-1)$.

In practice, learning curve estimates are useful to the extent that they can distinguish between substantially different possible true learning curve patterns. For estimating learning curve increments, with an increase in sample size from 50 to 150 (table 4, left columns), the range of possible true increments is roughly 0.05 (0.015 to 0.0684). The standard error for the BRIE estimate of these quantities is around 0.01, so the range of possible outcomes in our simulations is at least 5 times greater than the standard error. For estimating the learning curves themselves, the analogous results in table 6 (left columns) show a range of 0.08 in the true values, and a standard error of $0.05-0.07$. Thus the maximum observed difference is only slightly greater than the standard error, suggesting that the practical value of estimators of the learning curve may be limited, while useful information can be obtained from estimates of the learning curve increments. This point is underscored in the example considered in the next section. 


\begin{tabular}{cc|c|c|c||c|c|c|}
$p$ & $r$ & $\tau(150)$ & $\hat{\tau}_{B}(150), \mathrm{SD}$ & $\hat{\tau}_{S}(150), \mathrm{SD}$ & $\tau(200)$ & $\hat{\tau}_{B}(200), \mathrm{SD}$ & $\hat{\tau}_{S}(200), \mathrm{SD}$ \\
\hline 10 & 0.10 & 0.164 & $0.173,0.0647$ & $0.139,0.0911$ & 0.158 & $0.169,0.0651$ & $0.133,0.0945$ \\
15 & 0.10 & 0.149 & $0.153,0.0643$ & $0.113,0.0905$ & 0.141 & $0.146,0.0644$ & $0.107,0.0930$ \\
20 & 0.10 & 0.141 & $0.148,0.0676$ & $0.120,0.100$ & 0.133 & $0.139,0.0674$ & $0.112,0.103$ \\
25 & 0.10 & 0.143 & $0.155,0.0710$ & $0.130,0.111$ & 0.129 & $0.144,0.0707$ & $0.122,0.114$ \\
15 & 0.00 & 0.160 & $0.167,0.0670$ & $0.134,0.101$ & 0.151 & $0.161,0.0674$ & $0.127,0.103$ \\
15 & 0.25 & 0.131 & $0.137,0.0611$ & $0.121,0.116$ & 0.124 & $0.131,0.0613$ & $0.116,0.0917$ \\
15 & 0.50 & 0.106 & $0.101,0.0583$ & $0.919,0.0795$ & 0.101 & $0.105,0.0583$ & $0.0886,0.0811$ \\
15 & 0.75 & 0.0800 & $0.0761,0.0508$ & $0.0625,0.0550$ & 0.0761 & $0.0730,0.0508$ & $0.0597,0.0561$
\end{tabular}

Table 6: Mean and standard deviation of the BRIE and SUBEX estimator for estimating the learning curve when the training set size is increased from $n=50$ to $m=150$ and $m=200$. The BRIE estimator used a multivariate normal model with the restriction that $\Sigma_{i j}=\sigma^{2} \rho^{|i-j|}$ to estimate $p_{X}(x)$. The BRIE estimator is seen to be significantly less biased than the SUBEX estimator, as well as, possessing smaller standard deviation across training sets.

\section{Example: Predicting the four year survival proba- bility for CLL}

We next demonstrate the BRIE approach to estimating learning curves using data from a study of prognostic factors for outcomes of patients with chronic lymphocytic leukemia (CLL). The duration between the time that a subject entered a study and the time the subject required treatment (TTFT), a surrogate for disease progression, was obtained for 209 CLL subjects in a prospective study Ouillette et al. [2011]. For this analysis, the TTFT outcomes were dichotomized according to whether treatment was needed within four years of diagnosis. Eleven potential prognostic markers were used to predict this outcome using logistic regression. The markers are: ZAP70\%, p53 status, CD38\%, IgVH mutation status, age at diagnosis, Rai stage at diagnosis, number of positive lymph node groups, subchromosomal losses, chromosomal losses, subchromosomal gains, and chromosomal gains. This set of predictor variables includes binary (p53, IgVH), ordered categorical (Rai stage), count (chromosomal/subchromosomal losses and gains, positive lymph node groups), and continuous (ZAP70\%, CD38\%, age) measures. 
We calculated the BRIE estimator of the learning curves using two different models for the marginal distribution $p_{X}(x)$ of predictor variables. The first approach, which we denote "GM", used a Gaussian mixture. Here we stratified the training data into four groups, according to the joint pattern of values for the two binary variables (p53 and IgVH). Within each such group, the mean of the other nine non-binary variables was calculated, and a pooled covariance matrix for these nine variables over the four groups (centered at their respective means) was calculated. The GM model for the predictor variables was a four component Gaussian mixture, where the four components correspond to the four strata determined by the joint values of p53 and IgVH. The mixture components had means equal to the four stratum means in the training data, a common covariance structure equal to the pooled covariance matrix from the data, and marginal frequencies equal to the empirical frequencies of the four subgroups of training data defined by p53 and IgVH.

The second approach to modeling the covariate distribution, which we denote "GC", used a Gaussian copula. Here all data were converted to normal scores, then the correlation matrix of the normal scores was calculated. To produce simulated data from this model, we simulated Gaussian vectors according to this covariance matrix, then transformed each component of these vectors with the corresponding inverse normal scores function. The resulting model for simulated data has marginal distributions exactly equal to the univariate empirical distributions of the training set, and dependence which approximates the training set dependence.

The results are given in table 7. Four different training set sizes are used $(50,75,100$, 150). For each training set size $n$, we sampled $n$ observations without replacement from the overall CLL data set of 209 observations. These $n$ observations were used for three purposes: to estimate the misclassification error rate using cross-validated logistic regression, to fit a logistic regression model for $p_{Y \mid X}(y \mid x)$, and to fit models for the predictor variable distribution $p_{X}(x)$ using the GM and GC approaches. Together, $p_{X}(x)$ and $p_{Y \mid X}(y \mid x)$ were used to 
define the data-generating population $p_{Y, X}^{*}(y, x)=p_{Y \mid X}(y \mid x) p_{X}(x)$. We then generated data sets of size $n, 2 n$, and $3 n$ from $p_{Y, X}^{*}(y, x)$, fit another logistic regression model to each of these data sets, and evaluated the accuracy of these fitted rules relative to the data-generating population $p_{Y, X}^{*}(y, x)$. This process was repeated 1000 times and averaged to produce the results in table 7 .

Table 7 shows that improvement in prediction accuracy of only 2-3\% may occur when increasing the training set sample size by factors of 2-3 in this setting. This result is stable over the two approaches to modeling the predictor variables. As expected, the magnitudes of the gain are greatest for smaller training set sizes, and when the increment in training set size is larger (i.e. when comparing $\hat{\tau}_{I I}(3 n)-\hat{\tau}_{I I}(n)$ to $\left.\hat{\tau}_{I I}(2 n)-\hat{\tau}_{I I}(n)\right)$. Overall, this analysis suggests that only a small improvement in accuracy is likely to result from increasing the training set size in this setting. To achieve more substantial gains in accuracy, more informative markers, or a better modeling framework for these 11 markers should be sought.

As expected, $\hat{\tau}_{I I}$ underestimates the error rate, especially when the true error rate is high. This is a consequence of overfitting, since the strength of association in the fitted logistic regression model $\operatorname{logit} P(Y=1 \mid X=x)=\hat{\beta}^{\prime} x$ will tend to be stronger that the strength of association in the true model. In particular, if $Y$ and $X$ are independent in the true model, the fitted model will still have some association. As expected, this tendency diminishes as the sample size grows. As a result, $\hat{\tau}_{I I}(m)$ tends to increase with $m$, whereas the CV error, and presumably the true error, decrease with $n$. We note that for larger sample sizes, the copula model for $p_{X}(x)$ produces BRIE curve estimates that more closely resemble the cross-validation results.

As noted above, $\hat{\tau}_{I I}$ is generally too variable to be useful, so we focus on the accuracy gain, as shown in the final two columns of table 7. Since we have 209 data points to work with, we directly apply cross-validation on subsamples up to size 209 to provide a direct cross-validation based estimate that is known to be nearly unbiased. For example, under 


\begin{tabular}{|c|c|c|c|c|c|c|c|}
\hline & \multirow[b]{2}{*}{$n$} & \multirow[b]{2}{*}{$\mathrm{CV}$} & \multicolumn{3}{|c|}{$\hat{\tau}_{I I}$} & & \\
\hline & & & $n$ & $2 n$ & $3 n$ & & \\
\hline GM & 50 & 0.2775 & 0.2210 & 0.1968 & 0.1881 & 0.0242 & 0.0329 \\
\hline GM & 75 & 0.2636 & 0.2342 & 0.2157 & 0.2096 & 0.0185 & 0.0246 \\
\hline GM & 100 & 0.2580 & 0.2340 & 0.2201 & 0.2150 & 0.0139 & 0.0190 \\
\hline GM & 150 & 0.2363 & 0.2364 & 0.2264 & 0.2227 & 0.0100 & 0.0137 \\
\hline $\mathrm{GC}$ & 50 & 0.2775 & 0.2181 & 0.1922 & 0.1828 & 0.0259 & 0.0353 \\
\hline $\mathrm{GC}$ & 75 & 0.2636 & 0.2387 & 0.2196 & 0.2130 & 0.0191 & 0.0257 \\
\hline $\mathrm{GC}$ & 100 & 0.2580 & 0.2467 & 0.2316 & 0.2263 & 0.0151 & 0.0204 \\
\hline $\mathrm{GC}$ & 150 & 0.2363 & 0.2470 & 0.2358 & 0.2321 & 0.0112 & 0.0149 \\
\hline
\end{tabular}

Table 7: Learning curve analysis for the CLL data. Columns 7 and 8 show the gain in accuracy when the training set sample size increases from $n$ to $2 n$ (i.e. $\hat{\tau}_{I I}(2 n)-\hat{\tau}_{I I}(n)$ ), and from $n$ to $3 n$ (i.e. $\hat{\tau}_{I I}(3 n)-\hat{\tau}_{I I}(n)$ ), respectively.

the GC model, the error rate is predicted to drop from 0.2181 to 0.1828 when the training set sample size grows from 50 to 150, a gain of 0.0353. According to the cross-validation estimates, the gain is $0.2775-0.2363=0.0412$. Similarly, when the training set sample size grows from 75 to 150, the predicted gain in classification accuracy using the GC model for $p_{X}(x)$ is 0.0191 , and the corresponding estimate from cross-validation is 0.0273 .

\section{Discussion}

We have discussed three relatively simple approaches for estimating the learning curve of a classifier. SUBEX methods rely on a parametric model of the learning curve, and use unbiased estimates of the classification error rate for sample sizes smaller than the observed training set size to estimate the model parameters. IMPINT methods model the data distribution, from which the learning curve can be estimated at arbitrary sample sizes without the need to model the learning curve. 
Learning curve estimation is a challenging problem, and neither method considered here gives highly accurate results. However, we see that even in the limited range of settings considered here, gains in predictive performance ranging from 0.02 to 0.07 can be observed for three-fold increases in the training set size (table 4, column 2). For a problem where predictive accuracies in the 0.8-0.95 range are typical, knowing that a gain of 0.07 can be achieved may lead to a very different strategy for follow-up research compared to knowing that only a gain of 0.02 should be expected. The BRIE approach estimates the gain in predictive performance nearly unbiasedly, with a standard error of at most 0.01 . This provides us with power to confidently assess whether we are at the low end or the high end of the range of possible gains in performance.

The SUBEX and IMPINT approaches differ in several major ways, any of which could impact their performances. One potential drawback of the SUBEX approach is that the inverse power law model for $\tau$ may not be able to represent the true learning curve. An exact analytic expression for $\tau$ is unlikely to exist, necessitating the use of convenience parameterizations such as the inverse power law. Another concern for SUBEX is its use of cross-validation, which is known to have high variance (Efron [1983]). This variance may propagate to the learning curve estimate. The IMPINT method is not subject to these limitations, but concerns may arise about the need to estimate the data generating model, which is not necessary for the SUBEX approach. It is unclear which features of the data generating model are critical for learning curve estimation. At a minimum, the dimension and some measure of the strength of the predictive relationship are clearly relevant.

As noted above, the SUBEX approach models the learning curve, while the IMPINT approach models the full data distribution. The learning curve is a simpler object than the data distribution, hence SUBEX seems to require fewer assumptions. However, the learning curve is not directly observed. Any appropriate statistical modeling framework can be used to attain an estimate of the data generating model, and diagnostic and other tools are available 
to assess the fit of the model. Analogous tools for assessing the appropriateness of the learning curve model used by the SUBEX procedure are not readily available. Furthermore, the errors in the SUBEX procedure will be amplified by the need to extrapolate beyond the range of sample sizes that are directly estimated using subsampling. No analogous source of variation seems to be present in the IMPINT approach.

Learning curves have the potential to become a useful tool in applied statistics. One relevant analogy is to the widely-practiced fields of power analysis and sample size planning. In this setting, preliminary estimates of effect sizes are used to assess the power for various study designs. Learning curves can be viewed as a power analysis tool to be used when the research aims involve prediction, rather than focusing on estimation and hypothesis testing. As in classical power analysis, over-reliance on point estimates from small pilot studies may not be advised. In practice it would be advisable to consider a range of possibilities for key population parameters and attempt to delineate those situations where substantial gains in predictive performance are expected to occur.

\section{References}

S. Amari, N. Fujita, and S. Shinomoto. Four types of learning curves. Neural Computation, 4:605-618, 1992.

Leo Breiman and Philip Spector. Submodel selection and evaluation in regression. the $\mathrm{x}-$ random case. International Statistical Review, 60(3):291-319, 1992.

B. Efron. Estimating the error rate of a prediction rule: improvement on cross-validation. Journal of the American Statistical Association, pages 316-331, 1983.

Trevor Hastie, Robert Tibshirani, and Jerome H. Friedman. The Elements of Statistical Learning. Springer, 2009. 
David Haussler, Michael Kearns, H. Sebastian Seung, and Naftali Tishby. Rigorous learning curve bounds from statistical mechanics. Machine Learning, 25:195236, 1996.

T.R. Insel. Translating scientific opportunity into public health impact: a strategic plan for research on mental illness. Archives of General Psychiatry, 66(2):128, 2009.

S. Mukherjee, P. Tamayo, S. Rogers, R. Rifkin, A. Engle, C. Campbell, T.R. Golub, and J.P. Mesirov. Estimating dataset size requirements for classifying dna microarray data. Journal of Computational Biology, 10(2):119-142, 2003.

P. Ouillette, R. Collins, S. Shakhan, J. Li, E. Peres, L. Kujawski, M. Talpaz, M. Kaminski, C. Li, K. Shedden, and S.N. Malek. Acquired genomic copy number aberrations and survival in chronic lymphocytic leukemia. Blood, 118(11):3051-61, 2011.

Steven M. Snapinn and James D. Knoke. An evaluation of smoothed classification error-rate estimators. Technometrics, 27(2):199-206, 1985.

G. Toussaint. Bibliography on estimation of misclassification. IEEE Transactions on Information Theory, 20:472-479, 1974.

V.N. Vapnik. Estimation of Dependences Based on Empirical Data. Springer-Verlag, New York, 1982.

V.N. Vapnik and A.Y Chervonenkis. On the uniform convergence of relative frequencies of events to their probabilities. Theory of Probability and its Applications, 16:264280, 1971. 